\title{
e-Health interventions for healthy aging: a systematic review
}

\author{
Ronald Buyl ${ }^{1 *}$ (D, Idrissa Beogo ${ }^{2}$, Maaike Fobelets ${ }^{1}$, Carole Deletroz ${ }^{3}$, Philip Van Landuyt ${ }^{1}$, Samantha Dequanter',
} Ellen Gorus ${ }^{4}$, Anne Bourbonnais ${ }^{5,6}$, Anik Giguère ${ }^{7,8,9}$, Kathleen Lechasseur $^{10}$ and Marie-Pierre Gagnon ${ }^{8,9,10}$

\begin{abstract}
Background: Healthy aging (HA) is a contemporary challenge for population health worldwide. Electronic health (e-Health) interventions have the potential to support empowerment and education of adults aged 50 and over.

Objectives: To summarize evidence on the effectiveness of e-Health interventions on HA and explore how specific e-Health interventions and their characteristics effectively impact HA.

Methods: A systematic review was conducted based on the Cochrane Collaboration methods including any experimental study design published in French, Dutch, Spanish, and English from 2000 to 2018.

Results: Fourteen studies comparing various e-Health interventions to multiple components controls were included. The target population, type of interventions, and outcomes measured were very heterogeneous across studies; thus, a meta-analysis was not possible. However, effect estimates indicate that e-Health interventions could improve physical activity. Positive effects were also found for other healthy behaviors (e.g., healthy eating), psychological outcomes (e.g., memory), and clinical parameters (e.g., blood pressure). Given the low certainty of the evidence related to most outcomes, these results should be interpreted cautiously.
\end{abstract}

Conclusions: This systematic review found limited evidence supporting the effectiveness of e-Health interventions, although the majority of studies show positive effects of these interventions for improving physical activity in older adults. Thus, better quality evidence is needed regarding the effects of e-Health on the physiological, psychological, and social dimensions of HA.

Systematic review registration: The review protocol was registered in PROSPERO (registration number: CRD42016033163)

Keywords: e-Health intervention, Healthy aging, e-Health, Information technology

\section{Background}

Prospect studies foresee a worldwide growth of people aged over 60 years to at least 2 billion by 2050 [1]. More people are living longer and want to stay active and healthy to fully participate in life. However, decline in the biological, physiological, and cognitive systems inherent to

\footnotetext{
* Correspondence: ronald.buyl@vub.be

${ }^{1}$ Faculty of Medicine and Pharmacy, Department of Public Health Sciences, Biostatistics and Medical Informatics(BISI) Research Group, Vrije Universiteit Brussel (VUB), Brussels, Belgium

Full list of author information is available at the end of the article
}

aging may limit full social, cultural, and intellectual engagement in older persons [2]. Therefore, supportive strategies are needed to warrant a good quality of life. Healthy aging (HA) is defined as "the process of optimizing opportunities for physical, social and mental health to enable older people to take an active part in society without discrimination and to enjoy an independent and good quality of life" [3]. HA includes an active engagement with life, optimal cognitive and physical functioning, and low risk of disease that enables older people to participate within their limitations and continue to be physically, cognitively, 
socially, and spiritually active [4]. Ensuring HA for the population should be a priority in high-income countries today, but also in low-income countries that foresee aging of their population in the near future [5].

Worldwide, baby boomers are reaching the retirement age while policies are levied to keep older adults active in prolonging the working period (i.e., in Greece, France, Denmark) [6-8]. This cohort and onward generations in the "early old age" (defined by the WHO as people aged 50 years or above) [9] increasingly use information and communication technologies (ICT) in their daily activities [10]. With the rapid development of ICTs, which are getting more accessible and easier to use for these older adults, there is a huge potential to develop eHealth interventions targeting the growing population of 50 years and above. The WHO defines e-Health as the electronic exchange of health-related data collected or analyzed through electronic connectivity to improve the efficiency and effectiveness of healthcare delivery [11].

The rise in chronic conditions, which intensify in the last years of life, constitutes a contemporary challenge for health and welfare systems as it has profound implications for the planning and delivery of health and social care. A wide range of literature evidences that a longer life expectancy increases chronic health conditions and pressures the health system in terms of limited resources $[12,13]$ and public and private spending $[14,15]$. Various studies associate steadily spending growth to medical payment schemes [16] or merely to old age with comorbidities [15]. Nevertheless, the World Health Organization (WHO) Brasilia Declaration on Ageing in 1996 stated that "healthy older persons are a resource for their families, their communities and the economy" [17]. In view of these challenges and opportunities, following the United Nations members' meeting on Aging in Madrid in 2002, the WHO has proposed an active aging policy framework [18] and an age-friendly program plan in 2007 [9].

Among the interventions dedicated to maintain and improve older adults' active lifestyles and health, those incorporating e-Health receive increasing attention because of their potential to support empowerment and the recognition of their central role in today's society [19]. There are many examples of successful e-Health applications for health care and health promotion, such as telemedicine, electronic health records, virtual interventions, and personal health monitoring. With respect to HA, e-Health interventions offer older adults the opportunity to access health information and receive health and social care at home. These interactive interventions can empower, engage, and educate older adults [19].

In synthesizing the latest updates, Lattanzio et al. [20] highlight three main domains of development related to advances in technological innovation to support care: (1) disease management, (2) intelligent devices to improve autonomous living and mobility in older persons, and (3) specific needs for active aging. Among common eHealth interventions in support of HA, some are designed for virtual physical exercise [21, 22], and others promote networking [23], an active lifestyle [24], or independence [25]. Interestingly, recent studies contend a high intention to adopt e-Health interventions among older adults [26] and recognize these interventions to be relevant, adapted, and safe to use by these older users [27-30]. Furthermore, e-Health tools are designed to be more portable and lighter $[21,22]$. Other authors reported that they offer independence and confidence [27]. Nevertheless, despite technological developments and the multiplication of e-Health applications targeting older adults, knowledge on their effectiveness for supporting HA and its related outcomes has not been synthesized. There is an imperative to determine how eHealth can be used to improve old-age wellbeing.

Technologies that use ubiquitous computing and personalized algorithms play an important role in motivating people to adopt and maintain healthy behaviors as they age [31]. A systematic review of Web 2.0 interventions for chronic disease self-management in older adults found benefits on psychological outcomes as selfefficacy and quality of life, as well as on health behaviors (e.g., physical activity) [32]. Likewise, electronic games could offer huge opportunities for involving older people with cognitive and/or physical disabilities in activities that may support them to participate actively in everyday life [8]. A systematic review found some evidence regarding the effectiveness of exergames, digital gaming systems requiring physical exertion to play the game (e.g., Wii ${ }^{\text {tw }}$ games), in improving physical health in older persons [33]. Preschl et al.'s literature review of e-Health interventions targeting depression, anxiety disorders, and dementia in older adults found limited evidence of their effectiveness from high-quality studies, but promising results from smaller studies [34].

To date, to the best of our knowledge, there does not exist a systematic review that addresses the effectiveness of a range of e-Health interventions for supporting HA in all of its dimensions (e.g., physical, social, cognitive). Previous reviews [32-34] provide a starting point for a comprehensive systematic review that could map up current scientific evidence on e-Health interventions for HA.

\section{Objectives}

This systematic review intends to clarify the role of eHealth interventions in promoting HA among older adults. It targets two main objectives: (1) to identify and systematically summarize the best available evidence on the effectiveness of e-Health interventions on outcomes 
related to HA, as well as adverse effects related to these interventions, and (2) to explore how specific e-Health interventions (e.g., age-friendly, community interventions) and their characteristics (e.g., mode of implementation) may be implemented to effectively impact HA.

\section{Methods}

This systematic review was conducted based on the Cochrane Collaboration methods [35]. The review protocol was registered in PROSPERO (registration number: CRD42016033163). We used the PRISMA checklist [36] (see Supplementary file 1) to ensure reporting of all relevant information related to the systematic review. We also consulted the Synthesis Without Meta-analysis (SWiM) guidelines [37] to guide the use of alternative synthesis methods.

\section{Types of participants}

This review considered studies that include adults aged 50 or more (as 50 years is generally set as the beginning of the young old age) [9], living in the community or in institutional arrangement (e.g., nursing home). Exclusion criteria were as follows: (1) people with terminal illness, or (2) who are hospitalized, or (3) who have severe impaired cognition measured by specific tools such as the Mini Mental State Examination [38].

\section{Types of interventions}

e-Health interventions for healthy aging could include the following: Internet-based interventions, teleconsultations with health care providers, smartphone applications, interactive digital games, electronic records, and information systems. Types of interventions had to correspond to one of the seven technology focus areas proposed by the Center for Technology and Aging [39]. These areas are (1) medication optimization, (2) remote patient monitoring (RPM), (3) assistive technologies, (4) remote training and supervision (RTS), (5) disease management (DM), (6) cognitive fitness and assessment, and (7) social networking. eHealth interventions could take place at home, in a community health center, or another relevant setting. The interventions could be delivered individually or in groups and could take place over one or more sessions of various time frames. We excluded interventions that had an important face-to-face component; interventions that used conventional telephone, television or radio technologies, or technologies without an interactive component; and interventions targeted at treatment or prevention of complications of health problems.

\section{Types of comparisons}

The following comparisons were targeted: (1) any eHealth intervention versus usual service or practice (e.g., any service provided in the health care and/or social system, community, or individual initiative); (2) any eHealth intervention compared to any other e-Health intervention; (3) any e-Health intervention versus any other type of intervention (e.g., intervention with no or only minimal use of ICT); and (4) any e-Health intervention versus no intervention.

\section{Types of outcomes}

This review considered studies that include one or more of the following outcome measures as defined by the "Outcomes of interest to the Cochrane consumers \& communication review group" [40]. Primary outcomes related to HA included the following broad categories: (1) quality of life, including life satisfaction, wellbeing, activities of daily living, and leisure activities; (2) healthenhancing lifestyle, including physical activity, healthy diet, and alcohol and tobacco consumption; (3) motivation, including self-efficacy and self-esteem; and (4) social functioning.

Secondary outcomes included (1) knowledge, understanding, and skills acquisition; (2) decision-making including decision made and satisfaction with decision; (3) evaluation of care including goal attainment; (4) social support; and (5) any other behavior related to HA. This study also considered adverse effects related to e-Health interventions in the targeted population. Adverse effects could include social isolation, anxiety, and burden on informal caregivers.

\section{Types of studies}

We considered any experimental study design, including randomized controlled trials, non-randomized controlled trials, and quasi-experimental, before and after studies for inclusion.

Studies published from January 1, 2000, up to April 2018 in English, Dutch, French, or Spanish (languages spoken by team members) were considered for inclusion.

\section{Search strategy}

The search strategy included both published and unpublished studies through a three-step search strategy. An initial exploratory search in Medline and CINAHL was undertaken, as a test, to capture titles and abstracts, with the search equation that comprises common keywords, $\mathrm{MeSH}$ term-adapted to each database-and free vocabulary. Then, the results were analyzed to validate and built the final equation. Over this process, an information specialist of the Université Laval (MCL) validated the entire strategy. Finally, the validated search strategy was performed in the following databases: OvidMedline ${ }^{\circ}$ CINHAL, Cochrane Library, Embase, ERIC, Web of Science, PsycINFO, and Social Work Abstracts (see Supplementary file 2). The search for unpublished studies included clinical trial registers, conference 
proceedings, and an Internet search on Google and Google Scholar. Thanks to the reference manager Endnote, the research output of references was electronically rid of duplicates. Residual duplicates were manually removed. Finally, the reference list of all identified reports and articles was screened for additional studies.

\section{Study selection and data extraction}

All references were imported in the Rayyan reference screening system [41], and two team members (IB and PVL) independently screened all titles and abstracts for potential inclusion. Their results were combined, and a third reviewer (MPG) solved discrepancies. Thereafter, IB and PVL independently reviewed the full texts of preselected publications for inclusion. RB and MPG checked the list of included and excluded publications and solved discrepancies. IB and PVL performed data extraction independently, using a data extraction form in Excel, which documented details about the study objectives, used interventions, participants, study methods, and outcomes of significance to the review question and specific objectives. The results were compared and completed by RB and MPG.

\section{Quality appraisal}

Because all the selected studies were based on experimental designs, we employed the Cochrane Risk of Bias (ROB) tool for the assessment of possible methodological bias [35]. The reviewers independently rated the quality of each study as either "low," "unclear," or "high risk of bias." They took into consideration the seven domains of the ROB tool:

1) Sequence generation: describes the random components in the sequence generation of the study participants;

2) Allocation concealment: indicates how foreseeable the allocation of participants has proven to be;

3) Blinding of participants: assesses the measures employed to blind the study participants and personnel from knowing the intervention a participant would receive;

4) Blinding of outcome assessment: assesses whether the outcome assessors were blinded from knowing the intervention a participant would receive;

5) Incomplete outcome data: assesses whether the study participants withdrew from the analysis;

6) Selective outcome reporting: assesses a possible selection in expected or pre-specified outcomes, deriving from a systematic difference between reported and nonreported findings, based on the existence of a trial protocol and whether the expected outcomes have been reported in a pre-specified way.
7) Other sources of bias: includes the sample size and the power calculations of the trial that are based on the reported outcome or confounding.

\section{Data analysis and synthesis}

As the populations, interventions, and outcomes described in the included studies were heterogeneous, we were unable to pool quantitative data and conduct a meta-analysis. Therefore, we followed the guidelines regarding alternative forms for combining results [37].

\section{Results}

Figure 1 shows the study selection flow diagram. The initial search led to 7039 potentially relevant citations. After screening titles and abstracts, 60 publications were kept for further assessment, of which 10 articles were finally retained for the review [24, 42-50]. An updated search ran in May 2018 resulted in the inclusion of three additional peer-review publications [51-53] and one thesis [54].

In total, 21 peer-review articles and one thesisstemmed from 14 studies-were finally retained. The list of excluded publications and reasons for exclusion is provided in Appendix (Supplementary file 3).

The characteristics of the 14 included studies are presented in Table 1. Five studies were conducted in the USA [24, 43-45, 54], one in Japan [52], and the remaining eight in Europe [42, 46-51]. Among European studies, three were conducted in the Netherlands $[48,49,51]$, two in the UK $[47,53]$, and one in three countries (Greece, Spain, and Sweden) [42]. Furthermore, three studies presented different parts of their results in distinct publications: (i) Wijsman et al. [51, 62, 63], (ii) van het Reve et al. [50, 60, 61], and (iii) Peels et al. [48, 55-57].

When appraising the quality of the retained studies, we first noted that great variation existed with regard to sample size with a minimum of 14 and a maximum of 1729 participants. Seven studies had small samples $(n<$ 100) - for a total number of 3645 participants aged between 50 and 88 years old. Also, in the majority of studies, the samples comprised more women than men. Second, as shown in Fig. 2, the risk of bias was moderate to high across the studies, but the source of bias varied. The blinding (participants, personnel, or outcomes assessor) bias was present at a high risk or unclear in most studies. Sequence generation and allocation concealment were variable among the studies, which means that the potential selection biases were foreseeable in half of the studies. Finally, potential risk of bias related to incomplete outcome data and selective outcome was low in a large majority of studies, meaning that there was a low risk of reporting bias. We also noted the use of a wide range of validated questionnaires (such as quality of life: RAND 36; physical and psychological well-being: SF-36; 


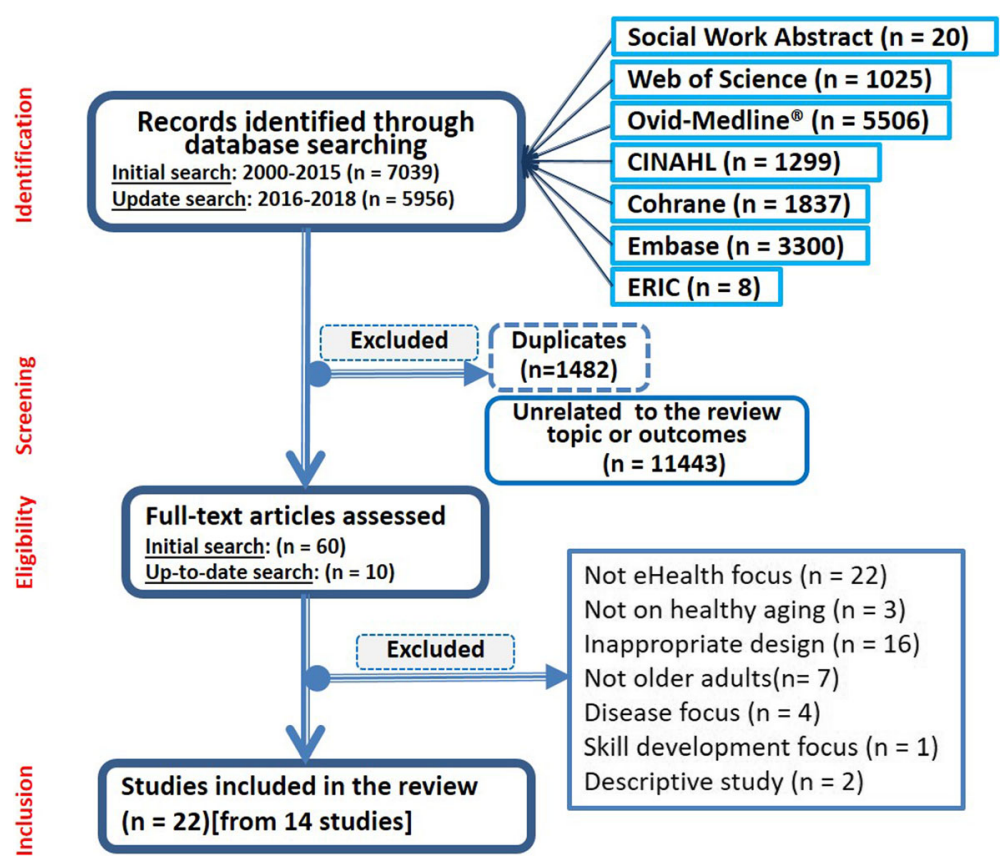

Fig. 1 PRISMA flow diagram illustrating the search strategy

wellbeing: SPF-IL scale) and non-validated rating scales (such as behavioral change self-assessment, IT literacy, engagement in activity) to assess the impact of the interventions.

\section{Focus area of the technology}

All the included study interventions were primarily Internet-based. These interventions were often compared with either paper-based interventions, interventions with a videophone component, mixed intervention, tailored or not. The technology devices that were part of the interventions consisted mainly of computers, tablets, or mobile phones. In reference to the Center for Technology and Aging classification [39], three areas are represented in this systematic review: remote patient monitoring, remote training and supervision, and social networking. One study consisted of an educational program with telemonitoring of step count, blood pressure, and body weight [52]. Most studies aimed to detect, train, and supervise patient remotely. One intervention was personalized with participants' information provided during the use of the webbased intervention [53], other interventions included information provision to increase daily physical activity $[50,51]$, or through a Web site with a tailored advice to undertake strength and balance training [47]. Finally, two studies evaluated social networking: one focused on Facebook and the use of an online diary [54], the second on an ICT-mediated social network [42].

As for the remaining studies, Cook et al. [24] focused more widely on health promotion goals (diet, physical activity, stress, tobacco use), whereas Slegers et al. [49] and van het Reve et al. [50] focused on computer training and Internet usage. Lastly, Homma et al. [52] focused on information technology literacy.

With respect to the outcomes, the majority of included studies (11/14) focused on physical activity (PA) $[24,43-48,50-53]$ with some focusing on the effect of physical activity on metabolic health and quality of life $[51,52]$ and another covering increasing healthy behavior [24]. The three other e-Health interventions targeted multiple dimensions including cognitive function, wellbeing, social engagement or connections, quality of life, or lifestyle modification [42, 49, 54].

\section{Effects of e-Health on healthy behavior outcomes}

The most often reported outcome in the included studies was physical activity (PA). Peels et al., comparing paper-based and web-based intervention on PA, concluded that the former was effective in increasing weekly days of sufficient PA $(p=0.005)$ at baseline and 6 months later $(p=0.042)$ [48]. In a similar vein, Irvine et al. showed that a web-based intervention to promote PA improved 13 of the 14 outcome measures and the intervention group maintained large gains on all 14 outcomes measured at 6 months [43]. In the Mouton 2015 study, a mixed intervention (center- and web-based intervention) led to improvement in PA level ( $p=$ $0.041)$, readiness for PA $(p=0.001)$, and improved awareness of PA ( $p=0.003)$ [46]. In a trial using text messaging, Kim and Glanz contended that motivational 


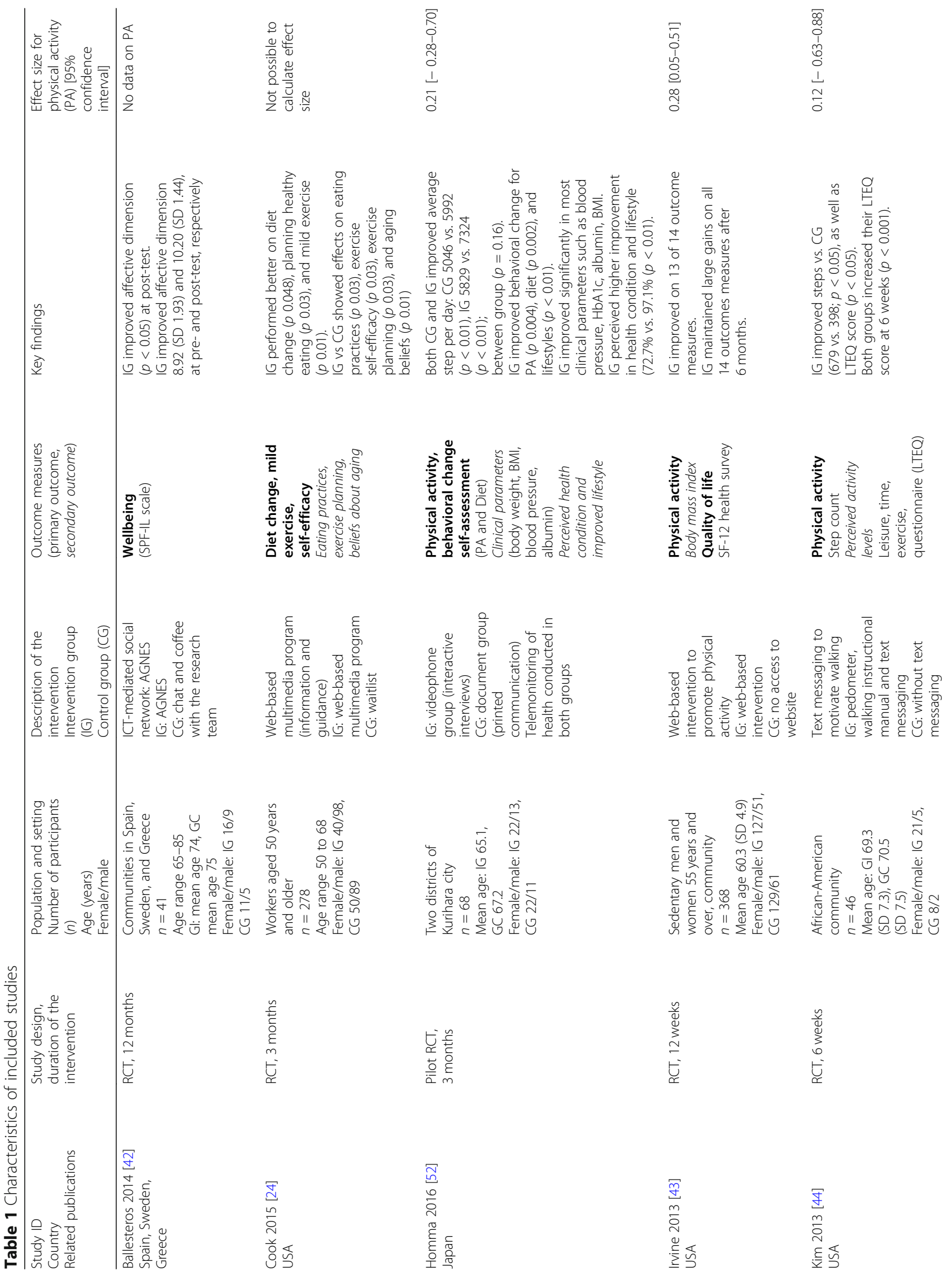




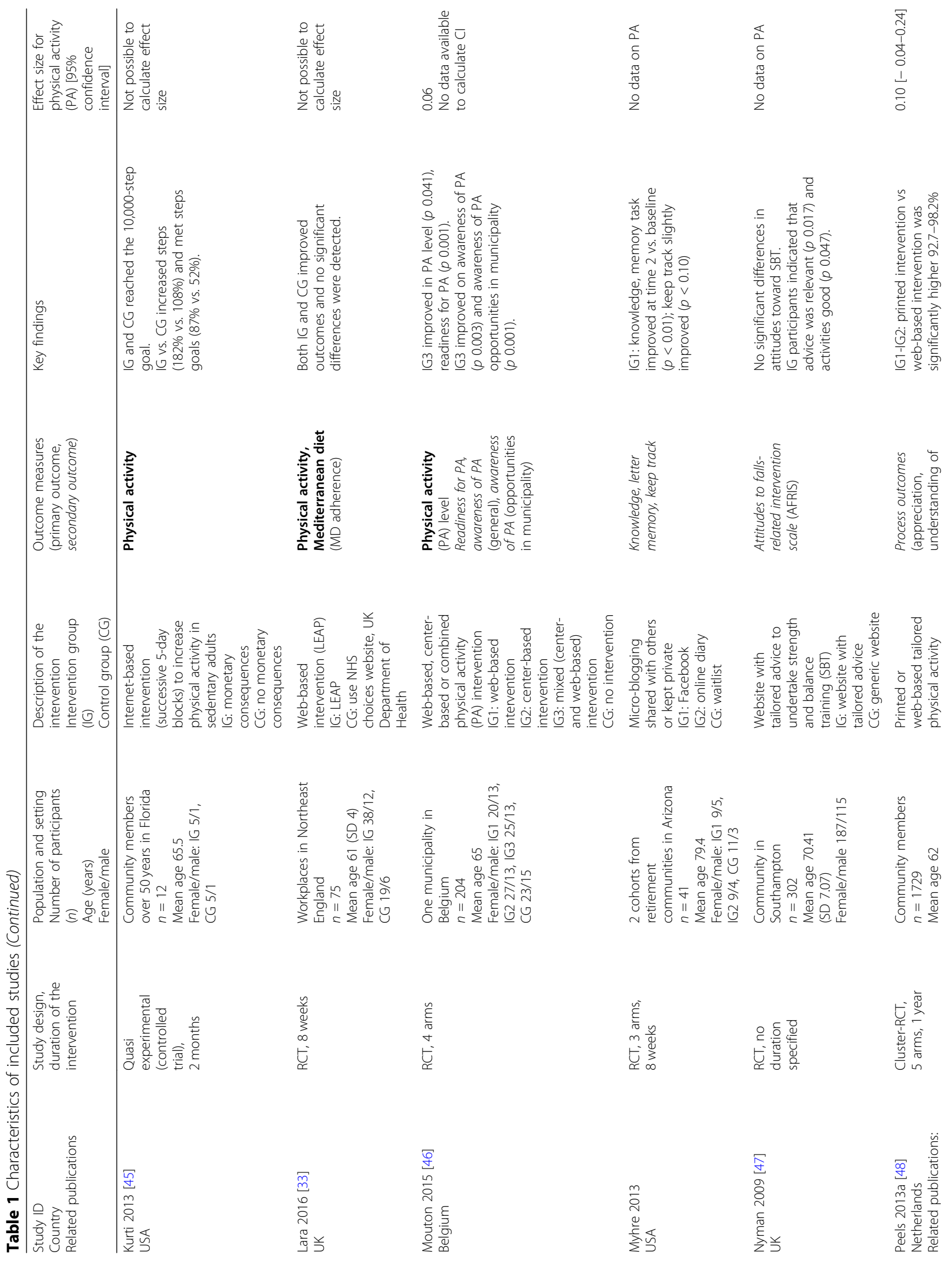




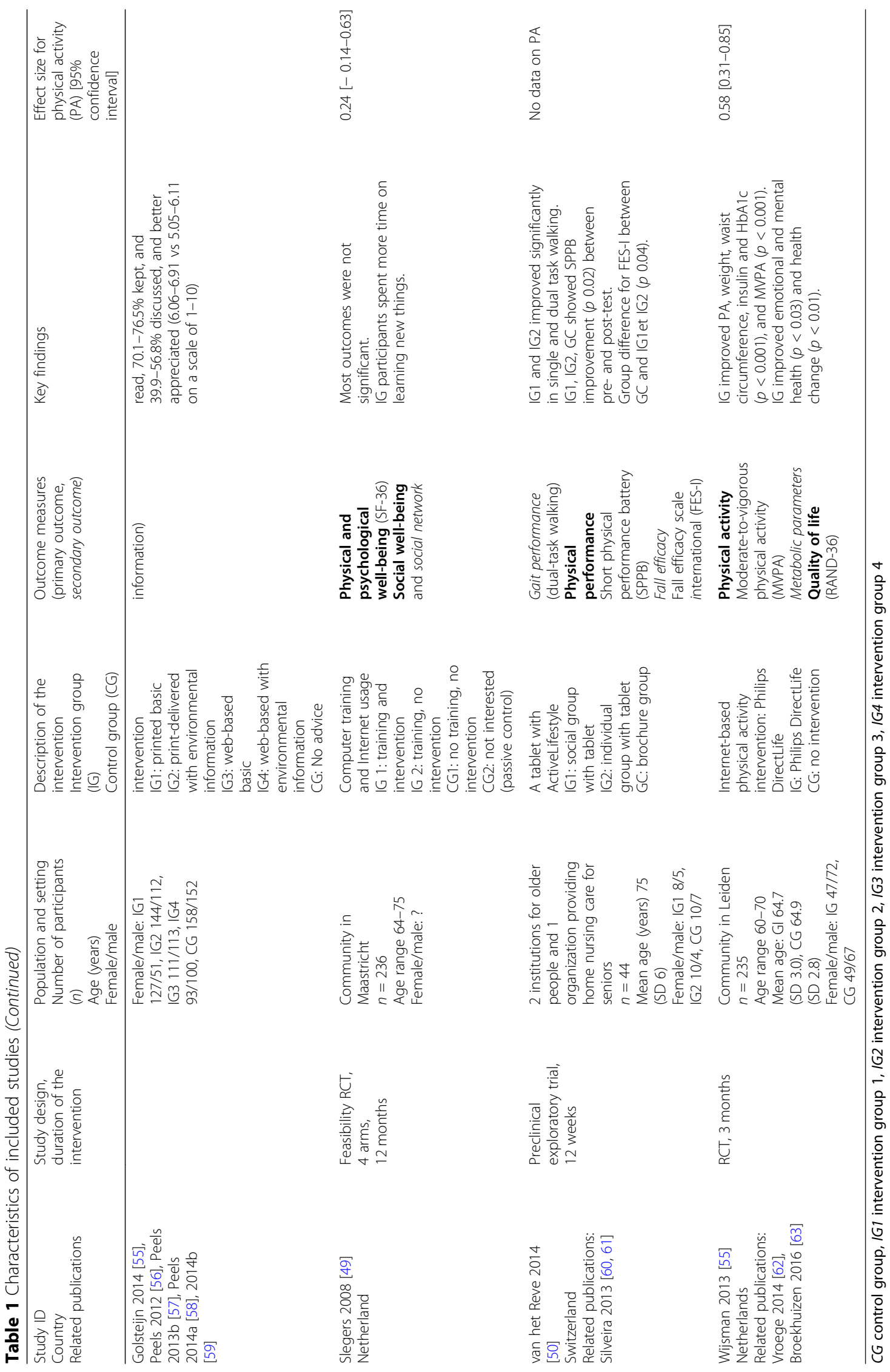




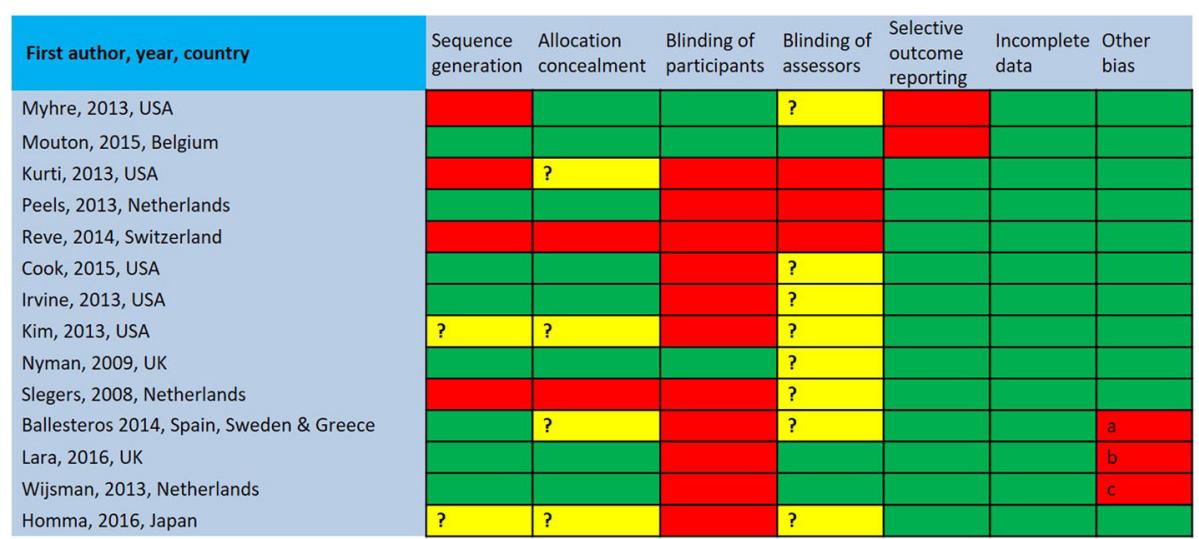

Fig. 2 Risk of bias assessment (Other bias: a volunteer, b reporting, c attrition)

text messaging (3 times/week) increases step count (679 vs. $398, p<0.05)$ as well as perceived activity level $(p<$ 0.05 ) [44]. Using a tablet intervention, van het Reve et al. [50] showed improvement in physical performance for all groups ( $p$ 0.02) compared to the brochure group in the single and dual-task walking $(p=0.03)$, as well as the falls efficacy $(p=0.04)$ [50]. Likewise, an Internetbased moderate-to-vigorous PA intervention of Wijsman et al. [51] led to a significant improvement of weight and waist circumference $(p=0.001)$. Finally, Homma et al. [52] reported an improvement in steps per day for both videophone intervention (interactive communication) and document groups $(p<0.01)$.

In a trial testing the addition of a monetary incentive to an Internet intervention, Kurti and Dallery concluded a higher percentage of goals achieved (87\%) in the group that received the monetary motivation [45]. Nevertheless, some studies were unable to find any significant difference in the PA outcomes targeted. For instance, Lara et al.'s pilot study showed weak and non-significant differences between both groups for PA [53]. However, we should not conclude in the absence of effect for this intervention, as the study was not sufficiently powered.

\section{Effects of e-Health on clinical parameter outcomes}

The study by Wijsman et al. comparing Internet-based PA intervention versus no intervention concluded to a significant improvement in clinical parameters, including insulin and HbA1c $(p<0.001)$; this is for moderate-to-vigorous PA $(p=0.001)$ [51]. Likewise, Homma et al. found significant improvements for blood pressure, HbA1c, and albumin when comparing the videophone intervention group to the document group [52].

\section{Effects of e-Health on psychological outcomes}

Regarding the psychological outcomes, in the Nyman et al. study [47], receiving a web-based tailored advice led to higher ratings of the advice relevance $(p=0.017)$ and goodness of fit of activities $(p=0.047)$. Besides, Wijsman et al. [51] demonstrated that the Internet-based PA intervention improved the emotional and mental health $(p=0.03)$ and health change $(p<0.01)$ in their measure of quality of life. In the Slegers et al. study, however, using computers and the Internet did not influence quality of life, well-being, and mood, nor the social network of healthy older individuals [49].

For their part, Ballesteros et al. found that an ICTmediated social network improved the affective dimension of wellbeing in their quality of life scale at post-test $(p<0.05)$ [42]. Similarly, Myhre et al.'s Facebook intervention improved knowledge $(p<0.01)$, as well as the Letter Memory task $(p<0.01)$ [54].

\section{Effects of e-Health on other outcomes}

Cook et al. [24] showed that their web-based multimedia program (information and guidance) had a significant effect on diet behavioral change self-efficacy $(p=0.05)$, planning healthy eating $(p=0.03)$, eating practices $(p=$ $0.03)$, exercise self-efficacy $(p=0.03)$, exercise planning ( $p$ $=0.03)$, and aging beliefs $(p=0.01)$. In the Peels et al. study [48], the process outcomes showed that the printed group significantly performed better in reading (92.798.2\%), keeping (70.1-76.5\%), and discussing (39.9-56.8\%) the advices received. Furthermore, the printed intervention was better appreciated than the web-based intervention (scores 6.06-6.91 versus 5.05-6.11, respectively, on a scale of 1-10) [48]. Moreover, Homma et al. [52] showed a significant positive change in self-assessment of PA ( $p=$ $0.004)$, diet $(p=0.002)$, and lifestyles $(p=0.005)$. Participant satisfaction using IT-related devices was significantly higher in the intervention (videophone) group than in the control group (printed documents) (40\% vs $15 \%$ ).

\section{Outcome synthesis and assessment of the certainty of the} evidence

Due to the important heterogeneity in the studies, it was not possible to conduct a meta-analysis for the outcomes 
of interest. However, following the SWiM guidance [37], we computed the effect estimates for PA as it was the most frequent outcome reported in the studies. Figure 3 shows the effect size and corresponding 95\% confidence interval (CI) for the studies that documented the effectiveness of e-Health on PA. However, some of these studies did not provide sufficient information to calculate the effect size $[24,53]$ or the CI $[46,50]$.

We assessed the certainty of the evidence based on the GRADE approach [64] considering the within-trial risk of bias, indirectness, heterogeneity, imprecision, and other considerations (Table 2). As it was not possible to pool the data for most outcomes, we considered the evidence provided by the individual trials as a whole to illustrate the level of evidence for each main category of outcomes. For all outcomes, the certainty of evidence is considered to be very low, mostly due to the risk of bias in individual trials and the imprecision of the estimates given the small sample sizes.

\section{Discussion}

The main objectives of this systematic review were to summarize the best available evidence on the effectiveness of e-Health interventions on HA and to explore how specific e-Health interventions may be implemented to effectively impact HA. To the best of our knowledge, this systematic review is the first to consider the overall effect of e-Health interventions on several dimensions related to healthy aging in older adults.

In this systematic review, we identified a broad variety of interventions that focused on promoting PA and other healthy behaviors, engaging in lifestyle change, and improving physical, psychological, and social wellbeing, which adds to existing literature [65-69]. Overall, most of the included studies were of moderate quality due either to their small sample size, the multiple-component nature of the interventions, their short duration, and the variable quality of the study designs [35].

For healthy older adults, our findings show positive effects of e-Health technology to promote healthy behaviors such as stimulating PA and awareness of PA, to enhance knowledge, and to facilitate behavior change and enhance psychological wellbeing. The use of the Center for Technology and Aging [39] classification system in our work enables the comparison of competing technologies. Furthermore, this classification used in telemedicine and e-Health fields may also facilitate communication among researchers, clinicians, and other users and target the specific technology's contribution to the health and wellbeing of older adults.

The provision of information was often at the core of the e-Health interventions. Still, there is a need to consider factors related to technology adoption by the older persons, such as interest in learning information and IT literacy [70]. In that line, Vaportzis et al. [71] warned

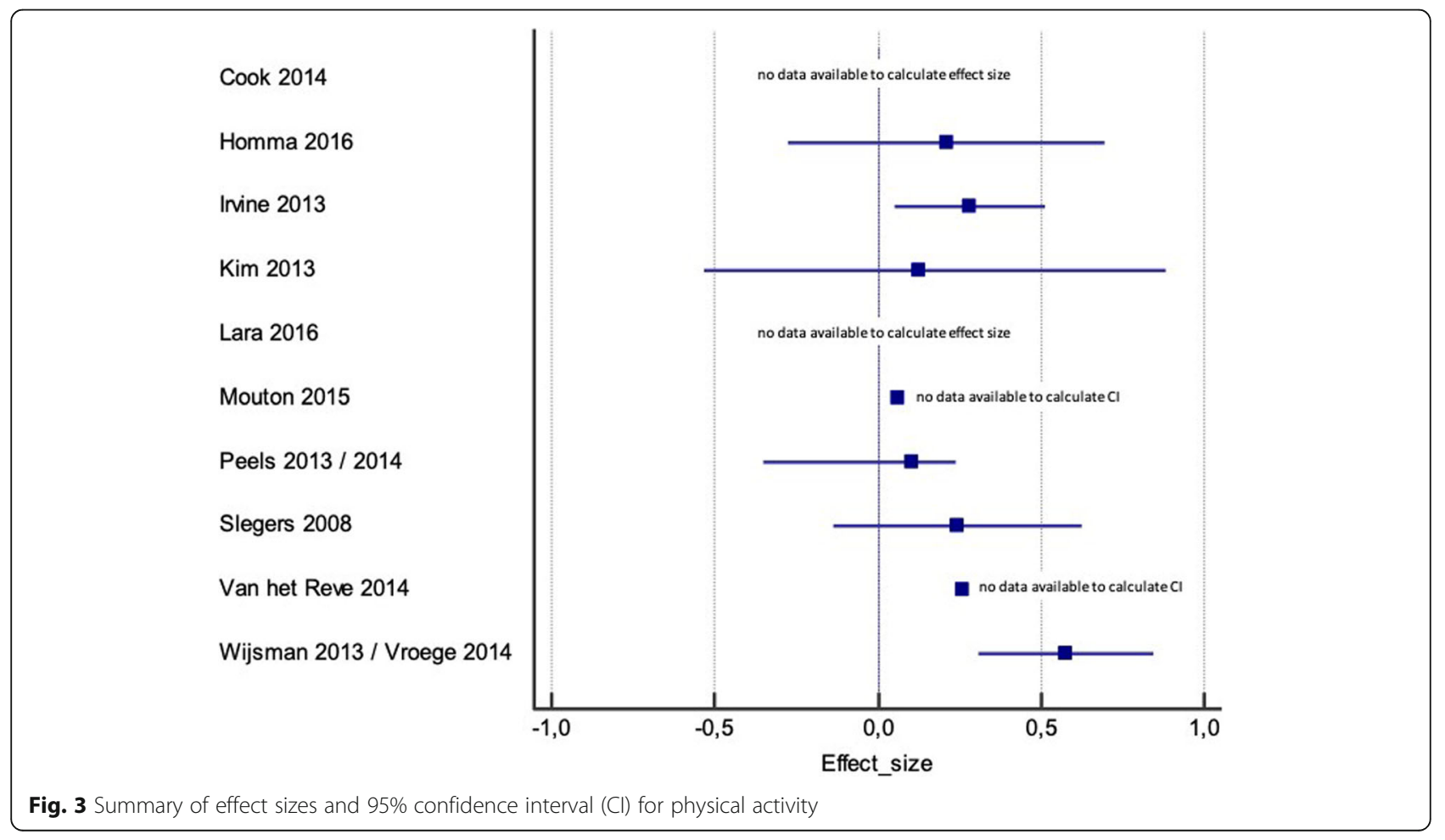


Table 2 Certainty of the evidence

\begin{tabular}{|c|c|c|c|c|c|c|c|}
\hline Outcome & $\begin{array}{l}\text { Number of } \\
\text { studies }\end{array}$ & $\begin{array}{l}\text { Risk of } \\
\text { bias }\end{array}$ & Inconsistency & Indirectness & Imprecision & $\begin{array}{l}\text { Other } \\
\text { considerations }\end{array}$ & Certainty \\
\hline Physical activity & 11 & Serious $^{a}$ & Not serious ${ }^{\mathrm{b}}$ & Serious $^{d}$ & Serious $^{f}$ & Serious $^{9}$ & $\begin{array}{l}\oplus 000 \\
\text { Very low }\end{array}$ \\
\hline Healthy eating & 2 & Serious ${ }^{a}$ & Serious ${ }^{c}$ & Serious $^{d}$ & Serious $^{\dagger}$ & Serious $^{9}$ & $\begin{array}{l}\oplus 000 \\
\text { Very low }\end{array}$ \\
\hline $\begin{array}{l}\text { Clinical parameters (body mass index, } \\
\text { HbAlc, cholesterol) }\end{array}$ & 5 & Serious $^{a}$ & Serious $^{c}$ & Serious $^{d}$ & Serious $^{f}$ & Serious $^{g}$ & $\begin{array}{l}\oplus 000 \\
\text { Very low }\end{array}$ \\
\hline Quality of life & 3 & Serious ${ }^{a}$ & Not serious ${ }^{\mathrm{b}}$ & $\begin{array}{l}\text { Not } \\
\text { serious }\end{array}$ & Serious $^{f}$ & Serious ${ }^{9}$ & $\begin{array}{l}\oplus 000 \\
\text { Very low }\end{array}$ \\
\hline Cognitive outcomes & 2 & Serious ${ }^{a}$ & Serious $^{c}$ & Serious $^{d}$ & Serious $^{f}$ & Serious $^{9}$ & $\begin{array}{l}\oplus 000 \\
\text { Very low }\end{array}$ \\
\hline $\begin{array}{l}\text { Psychological outcomes (wellbeing, depression, } \\
\text { loneliness) }\end{array}$ & 4 & Serious ${ }^{a}$ & Serious $^{c}$ & Serious $^{d}$ & Serious $^{f}$ & Serious $^{g}$ & $\begin{array}{l}\oplus 000 \\
\text { Very low }\end{array}$ \\
\hline $\begin{array}{l}\text { Social outcomes (social support, social } \\
\text { functioning) }\end{array}$ & 2 & Serious ${ }^{a}$ & Serious $^{c}$ & $\begin{array}{l}\text { Not } \\
\text { serious }^{d}\end{array}$ & Serious $^{f}$ & Serious $^{9}$ & $\begin{array}{l}\oplus 000 \\
\text { Very low }\end{array}$ \\
\hline
\end{tabular}

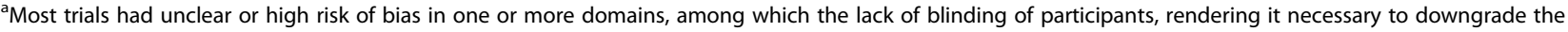
level of evidence

${ }^{b}$ Although there was some variation in the direction of effect, we did not downgrade the level of evidence since most outcomes showed a positive trend of the effectiveness of eHealth interventions

'For these outcomes, there was important heterogeneity in the measures used across studies

${ }^{\mathrm{d}}$ Most studies used surrogate outcome measures, among which self-reported measures of physical activity

${ }^{e}$ Quality of life was assessed directly using gold standard measures

fWhen confidence intervals were available, they were usually large. Also, most studies had modest sample sizes

${ }^{g}$ Given the limited number of included studies, we did not compute a funnel plot to check for publication bias, but it is likely that such bias is present given that

many studies have a modest sample size

about the following barriers related to IT adoption by older people: lack of instructions and knowledge, healthrelated barriers, cost, complexity of the technology, lack of social interaction, and communication. Furthermore, Hawley-Hague et al. [72] suggested to consider intrinsic factors related to older adults' attitudes around control, independence and perceived need/requirements for safety, and their motivation to use and go on using technologies. Several authors also identified some extrinsic factors, including usability, feedback gained, and costs, as important elements supporting older adults' attitudes and perceptions towards IT use [73, 74].

Other reviews have looked at the impact of ICT use by older people either on physical $[75,76]$ or on social dimensions [77, 78]. With respect to physical dimensions, the integrative review by Skjaeret et al. [75] found positive effects of exergaming on balance and gait and no major adverse effects. However, the number of included studies was low and most were of limited quality. Nevertheless, PA delivered through e-Health was found to improve adherence to exercise [76].

Chen and Shultz [77] found that ICT use was positively affecting social support, social connectedness, and social isolation among the elderly. However, the effect of ICT on loneliness was inconclusive, with some studies indicating a negative impact. $\mathrm{Li}$ et al. [78] reviewed the impact of exergames for older adults on social aspects and found generally positive impacts on loneliness, social connection, and attitudes towards others.

Although the evidence from our synthesis suggests that e-Health interventions are promising, we are still facing several challenges for large-scale implementation of these solutions among older adults. First, there is still a need to strengthen digital health literacy $[79,80]$. It is important to recognize that a lack of competence or limitation is often attributed to age-related cognitive decline [81, 82]. Critical competence is needed to effectively evaluate health information [83, 84]. Second, several methodological challenges remain for the evaluation of e-Health intervention. As e-Health interventions are at the intersection of biomedical, behavioral, computing, and engineering research, methods drawn from all these disciplines are required. Experimental designs such as RCTs to evaluate e-Health interventions are cost and time consuming, but remain important for demonstrating their effectiveness and cost-effectiveness $[11,85]$. Furthermore, Murray et al. recommend to undertake RCT only after ensuring that the intervention and its delivery package are stable, the intervention can be implemented with high fidelity, and when there is a reasonable likelihood that the overall benefits will be clinically meaningful [86]. Finally, the question of access to Internet arises. The digital divide [87] and the ongoing debate regarding the differences between users and nonusers of online health 
information among older adults [88], which could reinforce existing social differences, should be considered.

Although it provides a useful synthesis of the current evidence regarding e-Health interventions targeting healthy older adults, this review has some limitations. First, we excluded publications published in languages other than English, Dutch, Spanish, and French. This may have limited the scope of our investigation, but we consider that important international trials were captured by looking at the references of all included studies and searching manually in specialized journals. Second, an important limitation is related to our broad inclusion criteria, which led to include interventions that are quite heterogeneous. Our sample included participants from a wide age range -50 years old and above-thus some interventions might not be applicable to all age groups. This has limited a straightforward comparison and hindered a meta-analysis. However, the use of the Center for Technology and Aging [39] classification helped us to organize the results in a more coherent manner. Based on the types of technologies used and the nature of the interventions, it could be useful to promote a more structured taxonomy to present e-Health interventions in the literature, which could facilitate the identification of relevant studies and the aggregation of their results to inform decisions.

Finally, as the last search was conducted in April 2018, it is possible that more recent trials were not included. We conducted a rapid literature search in PubMed in March 2020 looking for potentially relevant studies published after the last update and identified one published study [89] and six published protocols [90-95], which indicates that several trials of e-Health interventions for $\mathrm{HA}$ are currently ongoing. We thus recommend to update this systematic review within the next 2 years as more evidence is likely to change the conclusions of the present systematic review.

\section{Conclusion}

This systematic review contributes to the evidencebase regarding the effectiveness of e-Health interventions in supporting HA. From our perspective, the critical question is how to best shape and direct our efforts to optimize the development and application of these technologies considering older adults' digital health literacy. As it is an emerging field, the evidence base on e-Health interventions for promoting $\mathrm{HA}$ is subject to quick evolution. The pace of technology development is rapid, and the technology could become obsolete at the time the results appear. Thus, innovative evaluation methods are needed to produce high-quality evidence in an appropriate timeframe in order to inform decisions regarding the implementation of effective technologies for HA.

\section{Supplementary information}

Supplementary information accompanies this paper at https://doi.org/10. 1186/s13643-020-01385-8.

Additional file 1: Supplementary file 1. PRISMA checklist.

Supplementary file $\mathbf{2}$. Search strategies. Supplementary file 3. List of excluded references and reasons.

\section{Abbreviations}

BMI: Body mass index; CTA: Center for Technology and Aging; DM: Disease management; HA: Healthy aging; ICT: Information and communication technologies; PA: Physical activity; PRISMA: Preferred Reporting Items for Systematic Reviews and Meta-Analyses; RCT: Randomized controlled trial; ROB: Risk of bias; RPM: Remote patient monitoring; RTS: Remote training and supervision; WHO: World Health Organization

\section{Acknowledgements}

We want to thank Ms. Marie-Claude Laferrière, an information specialist at Université Laval, for her support with bibliographic searches. We also acknowledge the contribution of Professor Sophie Éthier, School of Social Work, Université Laval, as co-investigator on the dissemination project.

\section{Authors' contributions}

$R B, M P G, A B, A G$, and $K L$ conceived the project and obtained funding. IB, $P V L$, and $C D$ did the study selection, data extraction, and data synthesis under the supervision of RB and MPG. IB and CD wrote the first version of the manuscript. MF, SD, and EG critically reviewed and completed the manuscript. All authors reviewed and approved the last version of the manuscript.

\section{Funding \\ This project was partly funded through a dissemination grant from the Canadian Institutes for Health Research.}

Availability of data and materials

All data generated or analyzed during this study are included in this published article and its supplementary information files.

Ethics approval and consent to participate

Not applicable

Consent for publication

Not applicable

Competing interests

The authors declare that they have no competing interests.

Author details

${ }^{1}$ Faculty of Medicine and Pharmacy, Department of Public Health Sciences, Biostatistics and Medical Informatics(BISI) Research Group, Vrije Universiteit Brussel (VUB), Brussels, Belgium. École des sciences infirmières et des études de la santé/School of Nursing and Health Studies, Université de Saint-Boniface, Winnipeg, Manitoba, Canada. ${ }^{3}$ School of Health Sciences (HESAV), University of Applied Sciences and Arts Western Switzerland, Avenue de Beaumont 21, CH-1011 Lausanne, Switzerland. ${ }^{4}$ Faculty of Medicine and Pharmacy, Department of Gerontology, Frailty in Ageing (FRIA) Research Group, Vrije Universiteit Brussel (VUB), Brussels, Belgium. ${ }^{5}$ Faculty of Nursing, Université de Montréal, Montreal, Quebec, Canada. ${ }^{6}$ Research Center of the Institut universitaire de gériatrie de Montréal (CRIUGM), Montreal, Quebec, Canada. ${ }^{7}$ Faculty of Medicine, Université Laval, Quebec, Canada. ${ }^{8}$ Centre de recherche sur les soins et services de première ligne de I'Université Laval (CERSSPL-UL), Quebec, Canada. ${ }^{9}$ Research Center of the Centre Hospitalier de Québec-Université Laval (CRCHUQ-UL), Quebec, Canada. ${ }^{10}$ Faculty of Nursing Sciences, Université Laval, Québec, 1050 avenue de la Médecine, Quebec, Canada. 
Received: 27 January 2020 Accepted: 11 May 2020

Published online: 03 June 2020

\section{References}

1. United Nations Population Fund. Ageing in the twenty-first century: UNFPA and HelpAge International, 2012. https://www.unfpa.org/sites/default/files/ pub-pdf/Ageing\%20report.pdf Accessed 15 Jan 2020.

2. Jin K, Simpkins JW, Ji X, Leis M, Stambler I. The critical need to promote research of aging and aging-related diseases to improve health and longevity of the elderly population. Aging Dis. 2015;6:1-5.

3. Swedish National Institute of Public Health. Healthy aging: a challenge for Europe. 2006.

4. Hansen-Kyle L. A concept analysis of healthy aging. Nurs Forum. 2005;40:45-57.

5. Henriquez-Camacho C, Losa J, Miranda JJ, Cheyne NE. Addressing healthy aging populations in developing countries: unlocking the opportunity of eHealth and mHealth. Emerg Themes Epidemiol. 2014;11:136.

6. Hofäcker D. In line or at odds with active ageing policies? Exploring patterns of retirement preferences in Europe. Ageing Soc. 2014;35:1529-56.

7. Hofäcker D, Naumann E. The emerging trend of work beyond retirement age in Germany. Increasing social inequality? Z Gerontol Geriatr. 2015;48:473-9.

8. Sixsmith A. Technology and the challenge of aging. In: US S, editor. Technologies for Active Aging; 2013. p. 7-25.

9. World Health Organization. Global age-friendly cities: a guide. 2007. https:// www.who.int/ageing/age_friendly_cities_guide/en/ Accessed 15 Jan 2020.

10. Pew Research Center. Older adults and technology use. 2014 http:// www.pewinternet.org/2014/04/03/older-adults-and-technology-use/. Accessed 15 Jan 2020.

11. World Health Organization. E-Health. 2016. https://www.who.int/ehealth/en/ Accessed 15 Jan 2020

12. Bähler C, Huber CA, Brüngger B, Reich O. Multimorbidity, health care utilization and costs in an elderly community-dwelling population: a claims data based observational study. BMC Health Serv Res. 2015;15:1.

13. Ha NT, Hendrie D, Moorin R. Impact of population ageing on the costs of hospitalisations for cardiovascular disease: a population-based data linkage study. BMC Health Serv Res. 2014;14:554.

14. Brinda EM, Kowal P, Attermann J, Enemark U. Health service use, out-ofpocket payments and catastrophic health expenditure among older people in India: the WHO Study on global AGEing and adult health (SAGE). J Epidemiol Community Health. 2015;69(5):489-94.

15. Islam MM, Yen L, Valderas JM, McRae IS. Out-of-pocket expenditure by Australian seniors with chronic disease: the effect of specific diseases and morbidity clusters. BMC Public Health. 2014;14:1-18.

16. Wubulihasimu P, Gheorghe M, Slobbe L, Polder J, van Baal P. Trends in Dutch hospital spending by age and disease 1994-2010. Health Policy. 2015; 119(3):316-23.

17. World Health Organization. Brasilia Declaration on Ageing. Brazil: World Health Organization, Ageing and Health Programme; 1996.

18. World Health Organization. Active ageing: a policy framework. 2002. https:// www.who.int/ageing/publications/active_ageing/en/ Accessed Jan 152020.

19. Hall AK, Stellefson M, Bernhardt JM. Healthy Aging 2.0: the potential of new media and technology. Prev Chronic Dis. 2012;9:110241.

20. Lattanzio F, Abbatecola A, Bevilacqua R, Chiatti C, Corsonello A, Rossi L, Bustacchini S, Bernabei R. Advanced technology care innovation for older people in Italy: necessity and opportunity to promote health and wellbeing. J Am Med Dir Assoc. 2014;15(7):457-66.

21. Miller KJ, Adair BS, Pearce AJ, Said CM, Ozanne E, Morris MM. Effectiveness and feasibility of virtual reality and gaming system use at home by older adults for enabling physical activity to improve health-related domains: a systematic review. Age Ageing. 2013;43:188-95.

22. $\mathrm{Wu} G$, Keyes LM. Group tele-exercise for improving balance in elders. Telemed e-Health. 2006;12(5):562-71.

23. Rébola C. Designed technologies for healthy aging: Morgan \& Claypool Publishers; 2015

24. Cook RF, Hersch RK, Schlossberg D, Leaf SL. A Web-based health promotion program for older workers: randomized controlled trial. J Med Internet Res. 2015;17:3.

25. Willner V, Schneider C, Feichtenschlager M. eHealth 2015 Special Issue: effects of an assistance service on the quality of life of elderly users. Appl Clin Inform. 2015;6(3):429-42.
26. Rosella LC, Fitzpatrick T, Wodchis WP, Calzavara A, Manson H, Goel V. Highcost health care users in Ontario, Canada- demographic, socio-economic, and health status characteristics. BMC Health Serv Res. 2014;14(532):1-13.

27. Brownsell S, Hawley MS. Automatic fall detectors and the fear of falling. J Telemed Telecare. 2004;10(5):262-6.

28. Heinbuchner B, Hautzinger M, Becker C, Pfeiffer K. Satisfaction and use of personal emergency response systems. Z Gerontol Geriatr. 2010;43(4):219-23.

29. Londei ST, Rousseau J, Ducharme F, St-Arnaud A, Meunier J, Saint-Arnaud J, Giroux F. An intelligent videomonitoring system for fall detection at home: perceptions of elderly people. J Telemed Telecare. 2009;15(8):383-90.

30. Mihailidis A, Cockburn A, Longley C, Boger J. The acceptability of home monitoring technology among community-dwelling older adults and baby boomers. Assist Technol. 2008:20:1-12.

31. Intille SS. A new research challenge: persuasive technology to motivate healthy aging. IEEE Trans Inf Technol Biomed. 2004;8:235-7.

32. Stellefson M, Chaney B, Barry AE, Chavarria E, Tennant B, Walsh-Childers $K_{\text {, }}$ Zagora J. Web 2.0 chronic disease self-management for older adults: a systematic review. J Med Internet Res. 2013;15:e35

33. Larsen $\mathrm{LH}$, Schou $\mathrm{L}$, Lund $H \mathrm{H}$, Langberg $H$. The physical effect of exergames in healthy elderly—a systematic review. Games Health. 2013;2:205-12.

34. Preschl B, Wagner B, Forstmeier S, Maercker A. E-Health interventions for depression, anxiety disorder, dementia, and other disorders in old age: a review. J Cyberther Rehabil. 2011:4:371-86.

35. Higgins JP, Green S. Cochrane handbook for systematic reviews of interventions (Vol. 4): Wiley; 2011

36. Liberati A, Altman DG, Tetzlaff J, et al. The PRISMA statement for reporting systematic reviews and meta-analyses of studies that evaluate healthcare interventions: explanation and elaboration. BMJ. 2009;339:b2700.

37. Campbell M, McKenzie JE, Sowden A, Katikireddi SV, Brennan SE, Ellis S, Hartmann-Boyce J, Ryan R, Shepperd S, Thomas J, Welch V, Thomson H. Synthesis without meta-analysis (SWiM) in systematic reviews: reporting guideline. BMJ. 2020;368:16890.

38. Folstein M, Folstein S, Mc Hugh P. Mini-mental state: a practical method for grading the cognitive state of patients for the clinician. J Psychiatr Res. 1975;12(3):189-98.

39. Center for Technology and Aging. Technologies to help older adults maintain independence: advancing technology adoption: Advancing Teachnology Adoption. http://www.phi.org/uploads/application/files/ begsbg0y101fcucuwhjnmndtv15r79rnfxjci0cppcwn2cnt67.pdf Accessed 15 Jan 2020

40. Cochrane Consumers in Communication Review Group. Outcomes of interest to the Cochrane consumers \& communication review group, 2012.

41. Ouzzani M, Hammady H, Fedorowicz Z, Elmagarmid A. Rayyan - a web and mobile app for systematic reviews. Syst Rev. 2016;5:210.

42. Ballesteros S, Toril P, Mayas J, Reales JM, Waterworth J. An ICT-mediated social network in support of successful ageing. Gerontechnology. 2014a; 13(1):37-46.

43. Irvine AB, Gelatt VA, Seeley JR, Macfarlane P, Gau JM. Web-based intervention to promote physical activity by sedentary older adults: randomized controlled trial. J Med Internet Res. 2013;15:2.

44. Kim BH, Glanz K. Text messaging to motivate walking in older African Americans: a randomized controlled trial. Am J Prev Med. 2013;44(1):71-5.

45. Kurti AN, Dallery J. Internet-based contingency management increases walking in sedentary adults. J Appl Behav Anal. 2013;46:568-81.

46. Mouton A, Cloes M. Efficacy of a web-based, center-based or combined physical activity intervention among older adults. Health Educ Res. 2015;30: 422-35.

47. Nyman SR, Yardley L. Web-site-based tailored advice to promote strength and balance training: an experimental evaluation. J Aging Phys Act. 2009;17: 210-22.

48. Peels D, De Vries H, Bolman C, Golsteijn R, Van Stralen M, Mudde A, Lechner L. Differences in the use and appreciation of a web-based or printed computer-tailored physical activity intervention for people aged over 50 years. Health Educ Res. 2013a;28(4):715-31.

49. Slegers K, Van Boxtel MP, Jolles J. Effects of computer training and Internet usage on the well-being and quality of life of older adults: a randomized, controlled study. J Gerontol Ser B Psychol Sci Soc Sci. 2008;63(3):176-84.

50. van het Reve E, Silveira P, Daniel F, Casati F, de Bruin ED. Tablet-based strength-balance training to motivate and improve adherence to exercise in independently living older people: part 2 of a phase II preclinical exploratory trial. J Med Internet Res. 2014;16(6):e159. 
51. Wijsman CA, Westendorp RG, Verhagen EA, Catt M, Slagboom PE, de Craen AJ, van der Ouderaa F. Effects of a web-based intervention on physical activity and metabolism in older adults: randomized controlled trial. J Med Internet Res. 2013;15:11.

52. Homma S, Imamura H, Nakamura T, Fujimura K, Ito $Y$, Maeda $Y$, Kaneko I. A comparative study on the effectiveness of one-way printed communication versus videophone interactive interviews on health promotion. J Telemed Telecare. 2016;22:56-63.

53. Lara J, O'Brien N, Godfrey A, Heaven B, Evans EH, Lloyd S, Rochester L. Pilot randomised controlled trial of a web-based intervention to promote healthy eating, physical activity and meaningful social connections compared with usual care control in people of retirement age recruited from workplaces. PLoS One. 2016;11(7):e0159703.

54. Myhre JW. Effects of online social networking on the cognitive, social, and emotional health of older adults: Doctoral thesis, University of Arizona; 2015.

55. Golsteijn RH, Peels DA, Evers SM, Bolman C, Mudde AN, de Vries H, Lechner L. Cost-effectiveness and cost-utility of a Web-based or print-delivered tailored intervention to promote physical activity among adults aged over fifty: an economic evaluation of the Active Plus intervention. Int J Behav Nutr Phys Act. 2014;11(1):122.

56. Peels DA, van Stralen MM, Bolman C, Golsteijn RH, de Vries H, Mudde AN, Lechner L. Development of web-based computer-tailored advice to promote physical activity among people older than 50 years. J Med Internet Res. 2012;14(2):e39.

57. Peels DA, Bolman C, Golsteijn RH, de Vries H, Mudde AN, van Stralen MM, Lechner L. Long-term efficacy of a printed or a Web-based tailored physical activity intervention among older adults. Int J Behav Nutr Phys Act. 2013;10(1):104.

58. Peels DA, Hoogenveen RR, Feenstra TL, Golsteijn RH, Bolman C, Mudde AN, Lechner L. Long-term health outcomes and cost-effectiveness of a computertailored physical activity intervention among people aged over fifty: modelling the results of a randomized controlled trial. BMC Public Health. 2014;14(1):1099.

59. Peels D, Van Stralen M, Bolman C, Golsteijn R, De Vries H, Mudde A, Lechner $L$. The differentiated effectiveness of a printed versus a Web-based tailored physical activity intervention among adults aged over 50. Health Educ Res. 2014;29(5):870-82.

60. Silveira P, van de Langenberg R, van het Reve E, Daniel F, Casati F, de Bruin, ED. Tablet-based strength-balance training to motivate and improve adherence to exercise in independently living older people: a phase ॥ preclinical exploratory trial. J Med Internet Res 2013;15(8):e159.

61. Silveira P, van het Reve E, Daniel F, Casati F, de Bruin ED. Motivating and assisting physical exercise in independently living older adults: a pilot study. Int J Med Inform. 2013;82(5):325-34

62. Vroege DP, Wijsman CA, Broekhuizen $K$, de Craen AJ, van Heemst D, van der Ouderaa FJ, Westendorp RG. Dose-response effects of a Web-based physical activity program on body composition and metabolic health in inactive older adults: additional analyses of a randomized controlled trial. Med Internet Res. 2014;16:12.

63. Broekhuizen K, de Gelder J, Wijsman CA, Wijsman LW, Westendorp RG, Verhagen $E$, van Heemst D. An internet-based physical activity intervention to improve quality of life of inactive older adults: a randomized controlled trial. J Med Internet Res. 2016;18:4.

64. Guyatt GH, Oxman AD, Schunemann HJ, Tugwell P, Knottnerus A. GRADE guidelines: a new series of articles in the Journal of Clinical Epidemiology. J Clin Epidemiol. 2011;64:380-2.

65. Aalbers T, Baars MAE, Rikkert MGMO. Characteristics of effective Internetmediated interventions to change lifestyle in people aged 50 and older: a systematic review. Ageing Res Rev. 2011;10(4):487-97.

66. Blazun H, Saranto K, Rissanen S. Impact of computer training courses on reduction of loneliness of older people in Finland and Slovenia. Comput Hum Behav. 2012;28(4):1202-12.

67. Changizi M, Kaveh MH. Effectiveness of the mHealth technology in improvement of healthy behaviors in an elderly population-a systematic review. MHealth. 2017;51.

68. Forsman AK, Nordmyr J. Psychosocial links between Internet use and mental health in later life: a systematic review of quantitative and qualitative evidence. J Appl Gerontol. 2017;36(12):1471-518.

69. Rocha NP, dos Santos MR, Cerqueira M, Queirós A. Mobile health to support ageing in place: a systematic review of reviews and meta-analyses. Int J EHealth Med Comm. 2019:1-21.

70. Boudin B. L'acceptation des nouvelles technologies par les personnes âgées [Elderly people acceptance of new technologies]. Lyon: Université Lumière Lyon; 2011. p. 2.
71. Vaportzis E, Giatsi Clausen M, Gow AJ. Older adults perceptions of technology and barriers to interacting with tablet computers: a focus group study. Front Psychol. 2017:8:1687.

72. Hawley-Hague H, Boulton E, Hall A, Pfeiffer K, Todd C. Older adults' perceptions of technologies aimed at falls prevention, detection or monitoring: a systematic review. Int J Med Inform. 2014;83:6.

73. Kappen DL, Mirza-Babaei P, Nacke LE. Older adults' physical activity and exergames: a systematic review. Int J Hum Comp Interac. 2018:1-28.

74. Vassli LT, Farshchian BA. Acceptance of health-related ICT among elderly people living in the community: a systematic review of qualitative evidence. Int J Hum Comp Interac. 2018;34(2):99-116.

75. Skjæret N, Nawaz A, Morat T, Schoene D, Helbostad JL, Vereijken B. Exercise and rehabilitation delivered through exergames in older adults: an integrative review of technologies, safety and efficacy. Int J Med Inform. 2016;85(1):1-16.

76. Valenzuela T, Okubo Y, Woodbury A, Lord SR, Delbaere K. Adherence to technology-based exercise programs in older adults: a systematic review. J Geriatr Phys Ther. 2018:41:49-61.

77. Chen YRR, Schulz PJ. The effect of information communication technology interventions on reducing social isolation in the elderly: a systematic review. J Med Internet Res. 2016;18(1):e18.

78. Li J, Erdt M, Chen L, Cao Y, Lee SQ, Theng YL. The social effects of exergames on older adults: systematic review and metric analysis. J Med Internet Res. 2018;20(6):e10486.

79. Eurobarometer. European citizens' digital health literacy. A report to the European Commission. 2014.

80. Norman CD, Skinner HA. eHealth literacy: essential skills for consumer health in a networked world. J Med Internet Res. 2006:8(2).

81. Kaphingst KA, Goodman MS, MacMillan WD, Carpenter CR, Griffey RT. Effect of cognitive dysfunction on the relationship between age and health literacy. Patient Educ Couns. 2014;95:218-25.

82. Wolf MS, Curtis LM, Wilson EA, Revelle W, Waite KR, Smith SG, Baker DW. Literacy, cognitive function, and health: results of the LitCog study. J Gen Intern Med. 2012;27(10):1300-7.

83. Chinn D, McCarthy C. All Aspects of Health Literacy Scale (AAHLS): developing a tool to measure functional, communicative and critical health literacy in primary healthcare settings. Patient Educ Couns. 2013; 90(2):247-53.

84. Diviani N, van den Putte B, Giani S, van Weert JC. Low health literacy and evaluation of online health information: a systematic review of the literature. J Med Internet Res. 2015;17(5):e112.

85. Kim H, Xie B. Health literacy in the eHealth era: a systematic review of the literature. Patient Educ Couns. 2017;100(6):1073-82.

86. Eysenbach G, CONSORT-EHEALTH Group. CONSORT-EHEALTH: improving and standardizing evaluation reports of Web-based and mobile health interventions. J Med Internet Res. 2011;13(4):e126.

87. Murray E, Hekler EB, Andersson G, Collins LM, Doherty A, Hollis C, Wyatt JC. Evaluating digital health interventions: key questions and approaches. Am J Prev Med. 2016;51(5):843-51.

88. Frennert S, Östlund BJD. What happens when seniors participate in new eHealth schemes? disability and rehabilitation. Assist Technol. 2016;11(7):572-80.

89. Muellmann S, Buck C, Voelcker-Rehage C, Bragina I, Lippke S, Meyer J, Peters M, Pischke CR. Effects of two web-based interventions promoting physical activity among older adults compared to a delayed intervention control group in Northwestern Germany: results of the PROMOTE community-based intervention trial. Prev Med Rep. 2019;15:100958

90. Alley S, van Uffelen JG, Schoeppe S, Parkinson L, Hunt S, Power D, Schneiders AG, Vandelanotte C. Efficacy of a computer-tailored web-based physical activity intervention using Fitbits for older adults: a randomised controlled trial protocol. BMJ Open. 2019;9(12):e033305.

91. Barbera M, Mangialasche F, Jongstra S, et al. Designing an Internet-based multidomain intervention for the prevention of cardiovascular disease and cognitive impairment in older adults: the HATICE trial. J Alzheimers Dis. 2018:62(2):649-63.

92. Pothier K, Soriano G, Lussier M, Naudin A, Costa N, Guyinnet S, Piau A Ousset PJ, Nourhashemi F, Vellas B, de Souto Barreto P. A web-based multidomain lifestyle intervention with connected devices for older adults: research protocol of the eMIND pilot randomized controlled trial. Aging Clin Exp Res. 2018;30(9):1127-35.

93. Recio-Rodríguez Jl, Lugones-Sanchez C, Agudo-Conde C, GonzalezSanchez J, Tamayo-Morales O, Gonzalez-Sanchez S, Fernandez-Alonso C, 
Maderuelo-Fernandez JA, Mora-Simon S, Gomez-Marcos MA, RodriguezSanchez E, Garcia-Ortiz L. Combined use of smartphone and smartband technology in the improvement of lifestyles in the adult population over 65 years: study protocol for a randomized clinical trial (EVIDENTAge study). BMC Geriatr. 2019;19(1):19.

94. van Vleet T, Voss M, Dabit S, Mitko A, DeGutis J. Randomized control trial of computer-based training targeting alertness in older adults: the ALERT trial protocol. BMC Psychol. 2018;6(1):22.

95. Vázquez FL, Torres ÁJ, Otero P, Blanco V, Lopez L, Garcia-Casal A, Arrojo M. Cognitive-behavioral intervention via interactive multimedia online video game for active aging: study protocol for a randomized controlled trial. Trials. 2019;20(1):692.

\section{Publisher's Note}

Springer Nature remains neutral with regard to jurisdictional claims in published maps and institutional affiliations.

Ready to submit your research? Choose BMC and benefit from:

- fast, convenient online submission

- thorough peer review by experienced researchers in your field

- rapid publication on acceptance

- support for research data, including large and complex data types

- gold Open Access which fosters wider collaboration and increased citations

- maximum visibility for your research: over $100 \mathrm{M}$ website views per year

At BMC, research is always in progress.

Learn more biomedcentral.com/submissions 